\title{
TEACHING PLC TIMERS AND COUNTERS PROGRAMMING USING MIT APP-INVENTOR
}

\author{
P. B. de Moura Oliveira ${ }^{1}$, J. Boaventura Cunha ${ }^{2}$, Filomena Soares ${ }^{3}$ \\ 1,2 UTAD-University of Trás-os-Montes and Alto Douro, ECT, 5000-801 Vila Real, Portugal \\ INESC TEC Technology and Science, Campus da FEUP, 4200 - 465 Porto, Portugal \\ ${ }^{3}$ Centro Algoritmi, School of Engineering, Universidade do Minho, 4800-058 Guimarães, Portugal \\ oliveira@utad.pt,jboavent@utad.pt,fsoares@dei.uminho.pt
}

\begin{abstract}
Current students and technologies demand using new learning/teaching techniques. The potentialities of using mobile devices such as smartphones for teaching/learning purposes are huge. However, in some teaching areas its use is still residual. The use of mobile applications in the context of teaching PLC programming techniques is addressed in this work. The MIT App-Inventor II is deployed to develop mobile applications for learning purposes. An android based application entitled Time-Counts is proposed here, developed to support the teaching/learning process of both Timers and Counters. Results regarding its use by students are presented.
\end{abstract}

Keywords: PLC Programming, MIT App Inventor, Timers, Counters, Control Engineering Education.

\section{Introduction}

The multitude of topics addressed in automatic control and industrial automation courses has been increasing significantly over the last decades. Indeed, within the Industry 4.0 [1] context, examples of emerging areas are: Internet of Things (IoT) [1,2], Smart manufacturing [3], Cyber-Physical Systems [4]. Within a 5-years engineering degree course, students must acquire knowledge and develop skills covering a wide range of industrial control subjects, such as: sensors and actuators, data acquisition, communication protocols, industrial machines, robotics, machine learning, decision support systems, supervisory control, etc. Many of these topics are covered in different and dedicated curricular units. However, given the multitude of topics the available in-class time for students to learn and develop more specialised skills is often insufficient. Thus, new teaching/learning techniques can be used advantageously to promote students out of the classroom autonomous learning.

Programmable logic controllers (PLC) and respective programming languages are mandatory topics within industrial automation courses. The main programming languages are: Ladder Diagrams (LD), (also commonly known as relay diagrams), Function Block Diagrams (FBD), Instruction List (IL), Structured Text (ST) and Sequential Function Charts (SFC). These languages are standardized by the norm IEC-61131-3 [5,6]. The functional block programming concept served latter as the basis of the IEC 61449 standard [7]. Two programming languages commonly addressed in PLC courses are
LD and SFC. The amount of in-class hours providing contact among teachers and students is often insufficient so students can properly apprehend PLC programming languages concepts. Thus, out of the class resources can be made available by teachers to students so they can remotely use them. The work reported here is focused in using new mobile devices to teach LD programming concepts, namely: Timers and Counters. It will be shown that PLC programming languages concepts can be provided to students through specific target made mobile applications.

Besides the lap-top computer, currently students use a wide range of mobile devices: tablets, smart phones, smart watches, etc. Indeed, mobile devices are part of university student's everyday life. Among these mobile devices, smartphones are exhaustively used by students, inside and outside the classroom. So, both teachers and students can take great advantages of using smart-phones to promote teaching and learning activities. Research about the use of mobile devices in students teaching and learning activities have been recently reported by [8]. With the ever-increasing Internet and communications progresses these devices can provide an efficient and flexible means to access information, anywhere and anytime, enabling a more personalized learning, which has been termed Mlearning $[8,9,10]$.

The use of smart-phones for teaching/learning activities requires the development of mobile applications. A highly desirable feature is the possibility of teachers and students to develop 
tailored made mobile applications to suit their particular interests. However, the development procedure of mobile applications can be an obstacle, particularly for teachers/students with no specific programming skills for developing such applications.

For Android operating system based devices, the MIT App Inventor 2 (MITApp2) [11] is a simple software tool which can provide solutions to empower both teachers and students in this domain [12,13]. MITApp2 uses a drag and drop methodology based on building blocks, avoiding the tedious process involved with syntax based programming languages. Indeed, users with elementary logic and programming concepts can easily accomplish to make their own applications.

MITApp2 has been previously used to provide students with applications addressing feedback control systems and industrial automation topics, as reported in [14] as well as to allow students to develop their own applications [15].

The idea, is to use MITApp2 to develop simple Apps addressing specific topics. An example of such an application entitled Time-Counts (TC) addressing Timers and Counters concepts was originally proposed in [16] addressing solely the timers component. As the TC App was significantly improved since, in this paper, TC functionalities covering both Timers and Counters are presented.

The selection of these two topics, Timers and Counters, as the bulk of the TC applications is based on their high relevance within programming languages for logic and sequential controllers. As it will be described, TC main idea is to perform simple simulations: i) for the three most used Timers: ondelay timer, TON, off-delay timer, TOF, and pulse timer, TP; ii) for the three most used Counters: count up counter (CTU), count down counter (CTD) and count up and down counter (CTUD). The general TC aim is to provide students, with a simple Android App to help them perceive timers and counters concepts, by running some simulations.

The rest of the paper is organized as follows: section 2 presents a brief description of the MITApp2 potential for developing apps for control engineering teaching/learning; Section 3 presents the TC application description for the timers components.

Section 4 presents the TC application description for the counters components. Section 5 presents some feedback of using TC by students and finally section 6 concludes the paper and outlines further work.

\section{MIT APP-Inventor to Teach/Learn Control Engineering}

Based on this paper authors experience by using the MITApp2 to develop simple Apps for teaching/learning purposes, some of the tool remarkable features are the following:

- It is easy to use by users with elementary programming skills;

- The time that goes from the App development stage to a ready to try application is very short;

- It is a freeware tool, with plenty of supporting information provided in the tool internet page, such as tutorials [18].

The application design process is organized in two parts; i) the graphical user interface and ii) the block behaviour programming. Both are based on a drag and drop approach. Figure 1 presents an example of the design used for an application within an UTAD University curricular unit of modelling and control systems (MCS_2015) in the first degree course of Biomedical Engineering. In this case several buttons are used in the main App screen to open other screens regarding the topics of Theory (Teoria), Problems (Problemas), Quizzes (Testes), etc.

Figure 2 presents a block programming example corresponding to the three buttons shown in Figure 1 , that when clicked opens the respective screen.

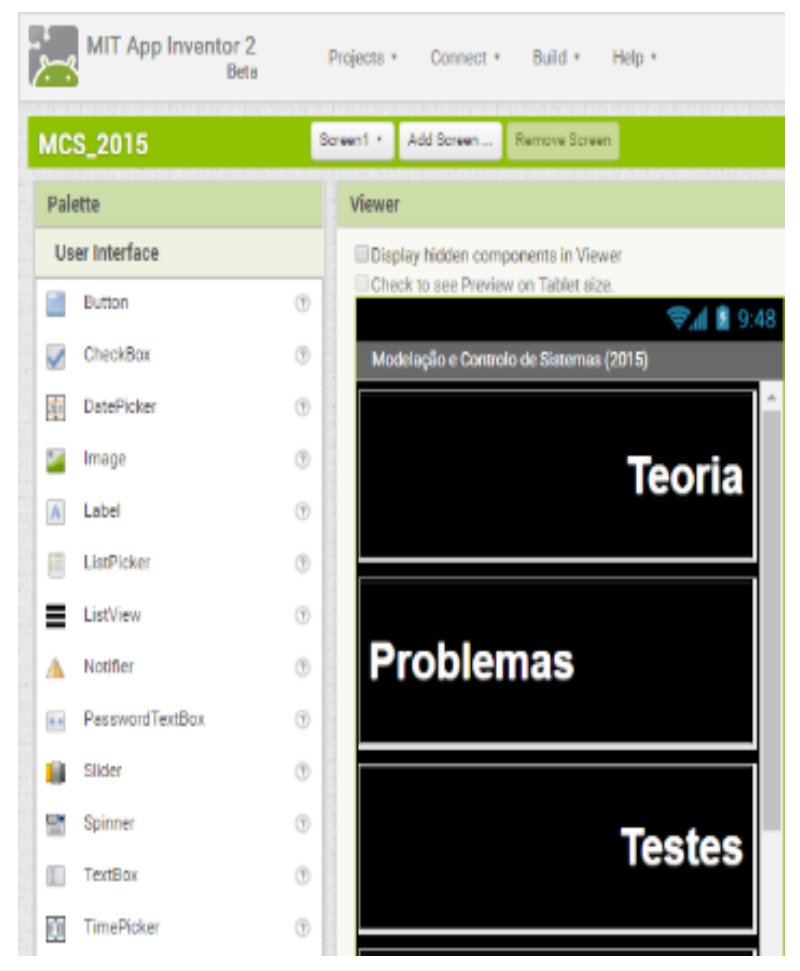

Figure 1: MITApp2 user interface design menu. 
MITApp2 enables to develop applications which can provide several useful features in the teaching/learning process, such as the following:

- information regarding specific theoretical topics (e.g. using a sliding show approach);

- problems to be solved;

- quizzes so they can test their learning regarding specific aspects, etc.

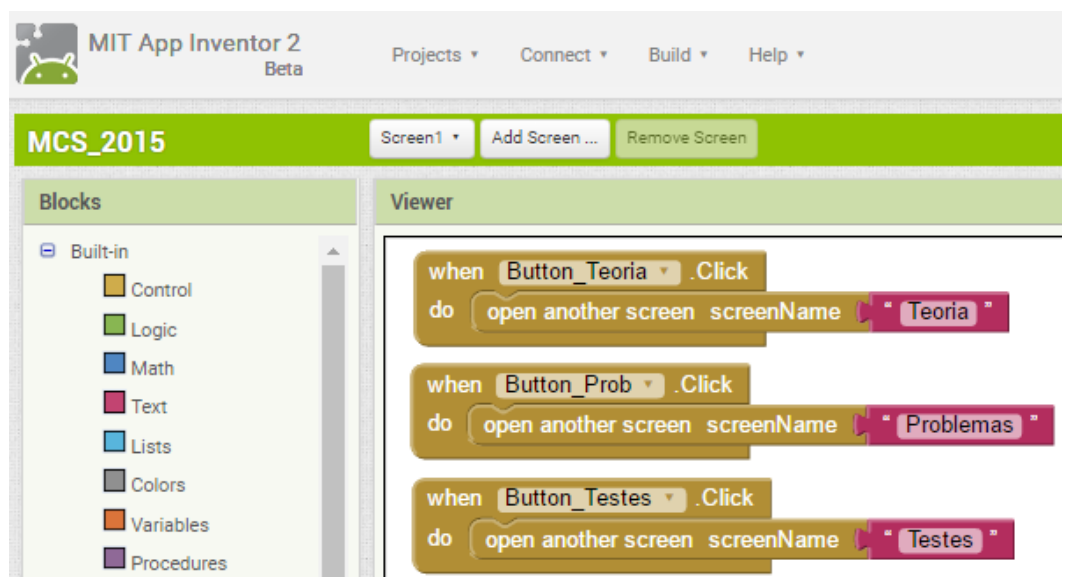

Figure 2: MITApp2 user interface design menu

Currently it is a common procedure for a lecturer to prepare slide show presentations for classes, made available to students via a learning management system. The general idea is that very soon the same procedure can also, as commonly, be used for mobile applications. Moreover, MITApp2 can also be used by students to perform projects and even to report assignment results, as published in [15].

\section{TimeCounts Application: Timers}

The TimeCounts (TC) application is organised in two main parts: Timers and Counters, with dedicated screen. The Timers covered are of the following type:

- On delay timer, TON,

- Off delay timer, TOF and

- Pulse delay timer, TP.

All three types of timers (TON, TOF and TP) can be represented with a generic block diagram as illustrated in Figure 3 a), where $I N$ represents the timer input, $Q$ the timer output, $P T$ the Preset time and ET the elapsed time. One of the TC learning objectives, is to enable students to simulate different possibilities used to control timers and then perceive what is supposed to occur to the timer output relating to the timer input and time variables. After the topic introduction in classes, students can use TC to understand timers operation and in practical classes, consolidate the learning process by replicating the simulation in the physical PLC. In this case, the SIEMENS S7-1200 [19] PLC is used in practical classes for the purpose. A pedagogical LD illustrative example is deployed for the different timers as illustrated generically in Figure $3 \mathrm{~b}$ ). It is an elementary LD with a single rung, using as the timer input a normally open switch, S1, and connecting to the timer output an output coil $L$.
As it will be explained, the TC App user will define the timer Preset time among several possibilities.

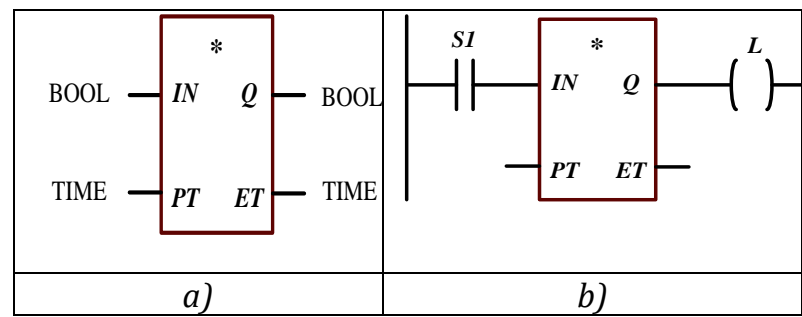

Figure 3: a) General block graphical representation for timers where * can be replaced by TON, TOF or TP;

b) General LD example used for Timers.

The TC App Timers menu screen is illustrated in Fig 4 a) with three interface buttons for the respective timer's types: TON, TOF and TP.

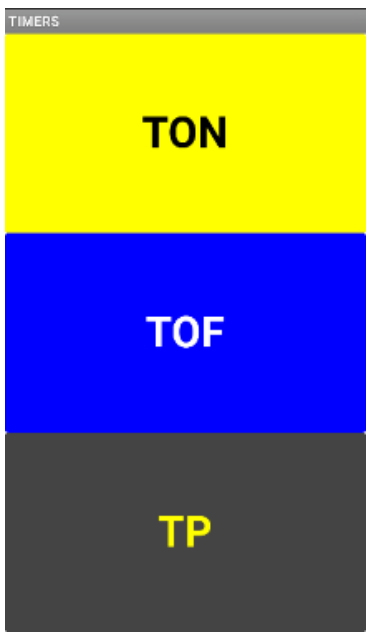

a)

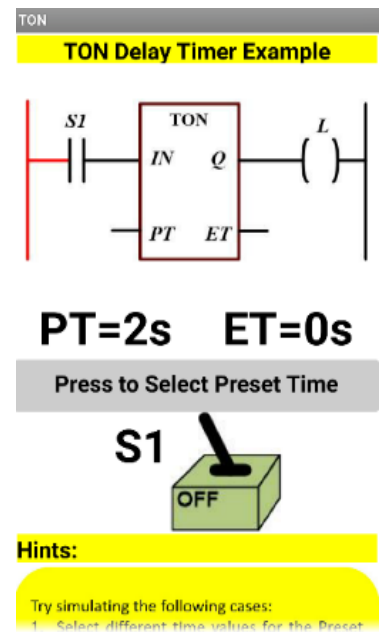

b)
Figure 4: a) TC Timers menu screen; b) TON delay timer example. 


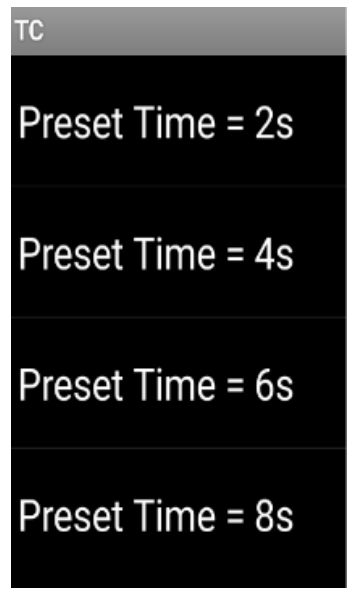

a)

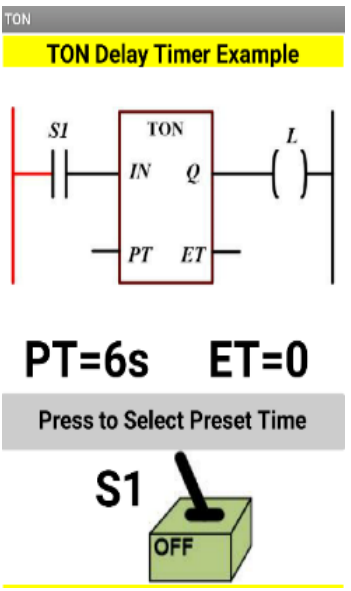

b)
Figure 5: a) Timers Preset time selection menu. b) TON example with PT=6sec and S1 in the off state.

Figure 4 b) presents a TON delay timer screenshot example. As it can be observed the normally open switch $S 1$ represents the logical state of the normally open contact $S 1$. The default Preset time is $P T=2$ sec. However it can be selected among several other options as illustrated in Fig 5 a).

Fig 6 a) illustrates the TON delay timer with the Preset time selected as $P T=6 \mathrm{sec}$, before $S 1$ is switched to the on state. The screenshot presented in Figure 6 a) shows the result of enabling the timer with $S 1$ on, while the $P T$ was not reached, and finally Figure $6 \mathrm{~b}$ ), corresponds to an instant after the $P T$ has been reached and $S 1$ was not switch off.

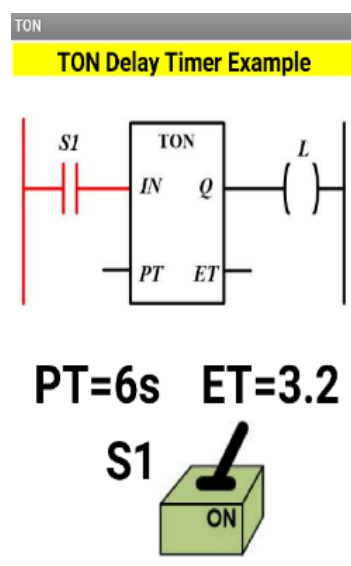

a)
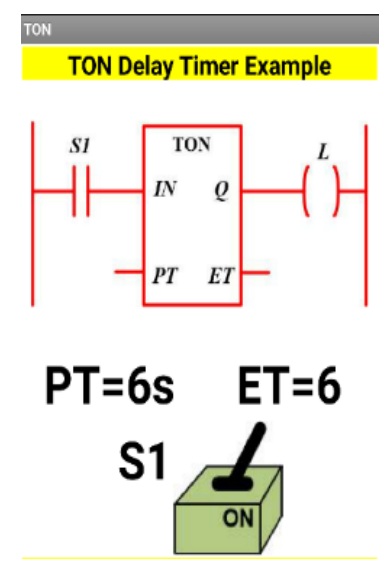

b)
Figure 6: a) TON running with switch on; b) TON when the PT has been reached and S1 is on;

It can be helpful to better perceive the different timers working principles to try to replicate chronograms such as the one presented in Figure 7, for the TON and TOF timer cases. Firstly by using the TC simulation and later on the real PLC. Thus, some exploratory hints are provided in the TC, for each timer. In the case of the TON timer some specific learning objectives are stated by the following questions:
- What happens to the timer output, $Q$, when the input changes to the on-state?

- How long must the input $I N$ remain in the on state in order to change the timer output $Q$ to the on state?

- After the output reaches the on state, how is the time period in which it remains on, controlled?

- What happens to the timer output, when the time period that input IN stays on is less than PT?

- What is the relation between the state of the output $Q$, and the activation of elements connected to $Q$ in the LD?

A very interesting and relevant practical issue regarding the TON timer is the adequate selection of the contact to be used as the IN input. Frequently, in practical classes, student use as TON input sensors logic state (with positive logic), which are in the on state, for brief instants (e.g. the detection of components passing in a conveyor belt). This causes the TON timer to start counting the Preset time, but as the input is switched off much before the PT is reached (see case illustrated by $t_{1}$ in Figure $7 \mathrm{a}$ ), the timer output is never activated. One way to avoid this problem is by memorising the event occurrence detected by the sensor, using a PLC binary memory register, and then use the corresponding contact as TON input. In this way the timer input is controlled by the PLC programmer, ensuring the activation of its output, as well as the period that it stays in the on state.

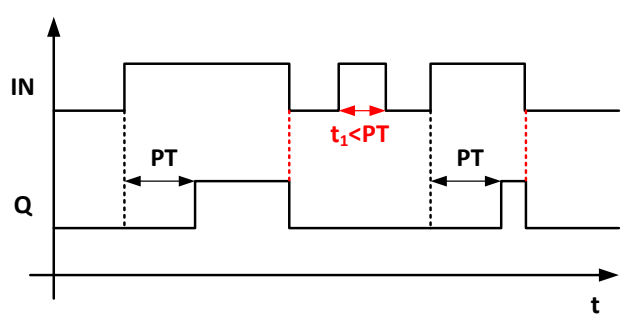

a)

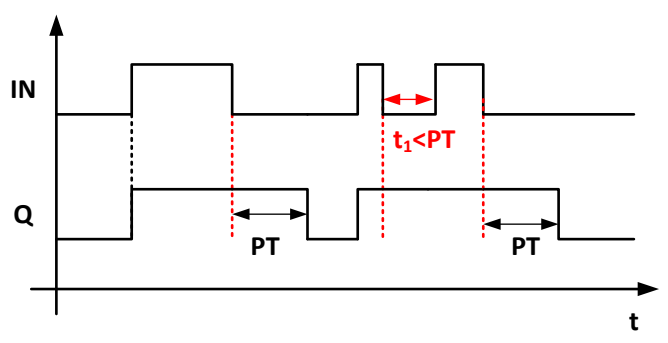

b)

Figure 7: Chronogram example for a) TON Timer; b) TOF Timer.

Fig 8 a) presents a TC screenshot, regarding a TOF timer simulation, where it can be seen that the timer output is on since the timer input is on. In this case $E T$ will begin to count as soon as the switch $S 1$ is turn off (see example in Fig 8 b)). 


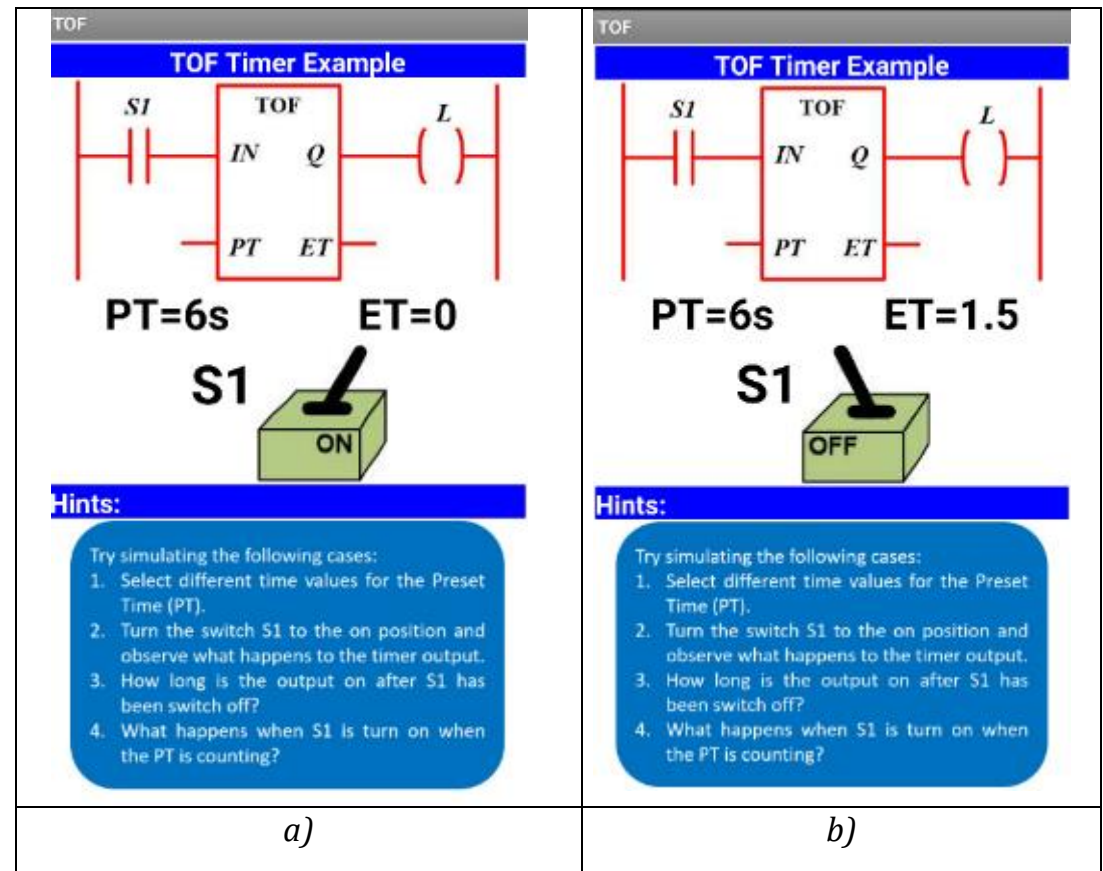

Figure 8: a) TOF example: timer output is on as soon as $S 1$ is switched on b) TOF example: the PT time is being counted since $S 1$ was switched off.

Based on the chronogram example provided in the App and presented in Fig 7 b), some TOF timer learning objectives are stated by the following questions:

-What happen to the timer output, $Q$, when the $I N$ input changes to the on state?

- When does the Preset time $P T$ starts to be counted?

- How long must the input $I N$ remain on in order to change the timer output $Q$ to the on state?
- What happens to the timer output, when the input IN passes from the on to the off state, and changes again to the on state before the $P T$ is reached (example represented as $t 1$ in Fig 7 b))?

- What is the relation between the output $Q$ state, and the activation of elements connected to $Q$ in the $L D$ ?

Figure 9 presents TC screenshots for a TP timer simulation before the switch $S 1$ changes to the on state (Figure 9 a)), and when the PT is counting (Figure $9 \mathrm{~b}$ )).

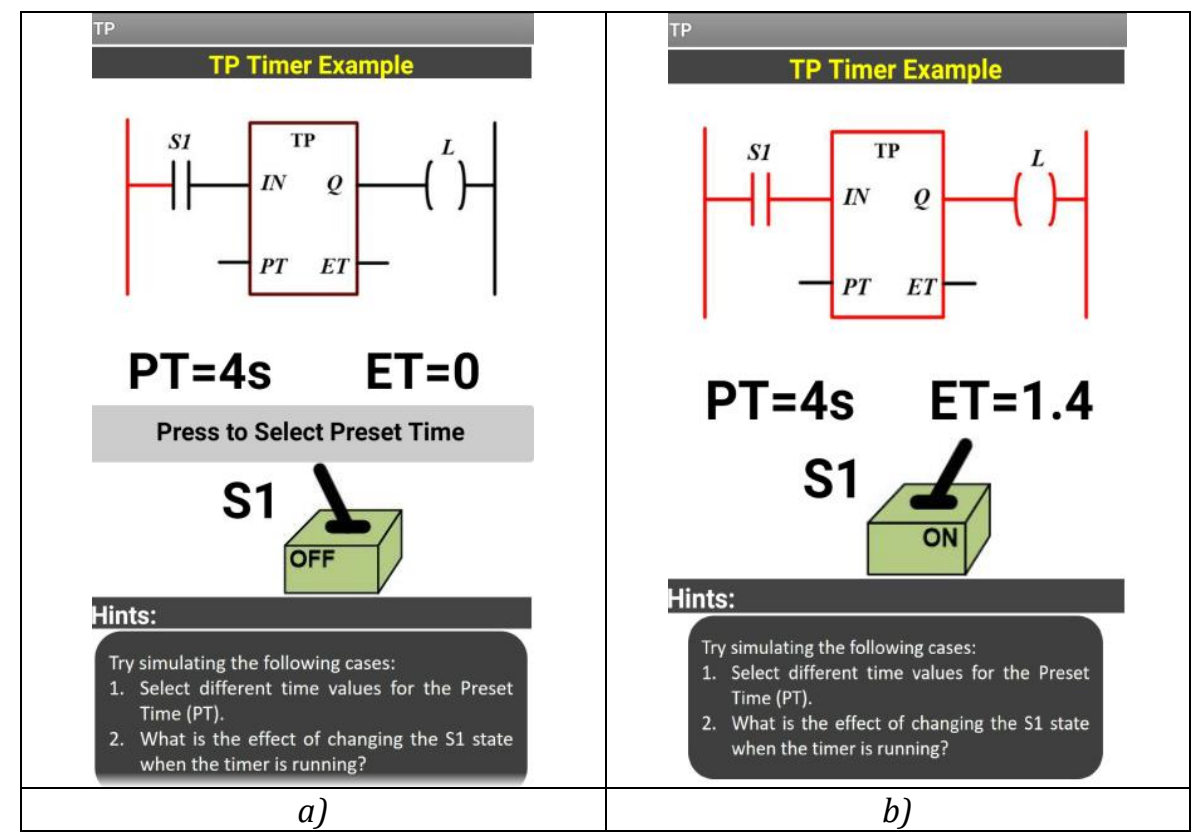

Figure 9: TP example: a) before S1 is switch on. b) After S1 has been switched on and the PT has not been reached. 
Based on the chronogram example provided in the App and presented in Figure 10, some specific TP learning objectives are stated by the following questions:

- What happens to the timer output, Q, when the input changes to the on-state?

- When does the Preset time PT starts to be counted?

- Does the Input $I N$ has to remain on when the PT time is been counted (see $t 1$ case in Figure 10)?

- What is the relation between the output $Q$ state, and the activation of elements connected to $\mathrm{Q}$ in the LD?

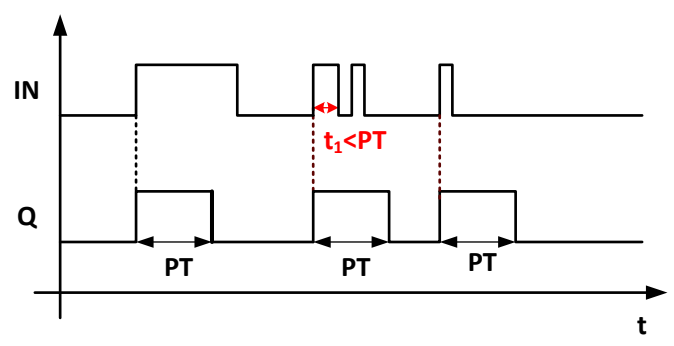

Figure 10: Chronogram example for TP Timer.

\section{TimeCounts Application: Counters}

The Counters covered are of the following type:

- Count up counter, CTU,

- Count down counter, CTD and

- Count up and down counter, CTUD.

The CTU counter can be represented with a generic block diagram as illustrated in Figure 11 a), where $C U$ represents the increment counter input, $Q$ the timer output, $R$ the Reset input, $P V$ the Preset value and $C V$ the Counted value. A simple LD illustrative

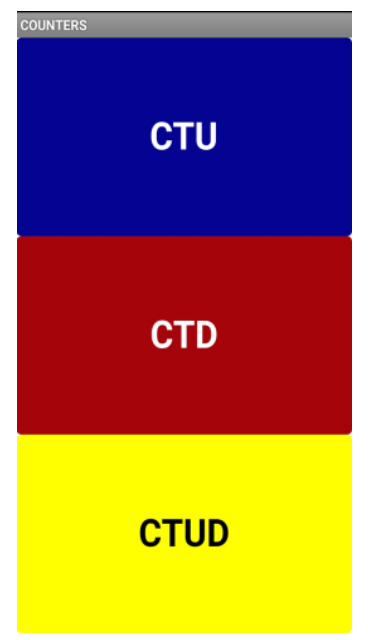

a) example is deployed for the CTU counter as illustrated generically in Figure 11 b). It is an elementary LD with a single rung, using as the: counter input and a normally open switch, S1; reset input a normally open switch, $S 2$ and connecting to the counter output an output coil, $L$.

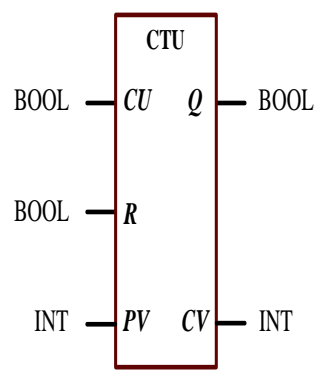

a)

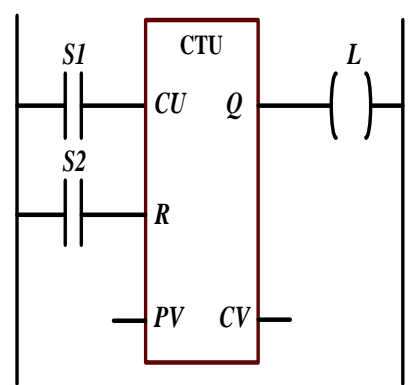

b)
Figure 11: a) General block graphical representation for CTU counter; b) General LD example used for CTU.

The TC App Counters menu screen is illustrated in Fig 12 a) with three interface buttons for the respective timer's types: CTU, CTD and CTUD.

Figure 12 b) presents the CTU counter example. As it can be observed the normally open switch $S 1$ represents the logical state of the normally open contact $S 1$. A Preset value should be selected first, which in this example was set to $C V=6$. Every time $S 1$ changes its state from off to on state the Counted value (CV) is increased.

The case when the $\mathrm{PV}$ is reached by the $\mathrm{CV}$ is presented in Figure 13 a). The reset of the $\mathrm{CV}$ is illustrated by screenshot presented in Figure $13 \mathrm{~b}$ )

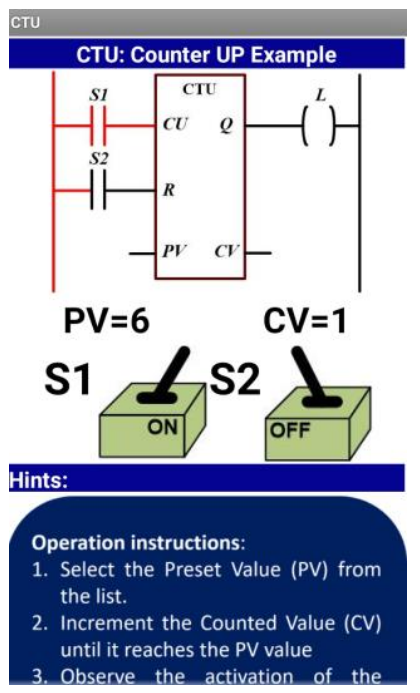

b)

Figure 12: CTU screenshots: a) TC Counters menu screen; b) Preset value selected and S1 activated.

A chronogram example for the CTU counter is presented in Figure 14.
Students can follow this example by simulating the pulse sequence counting. 
Some CTU specific learning objectives are stated by the following questions:

- What happens to the timer output, $Q$, when the $C V$ reaches the $P V$ ?

- What happens to the $C V$ when the counter input $C U$ is activated after the counter output,

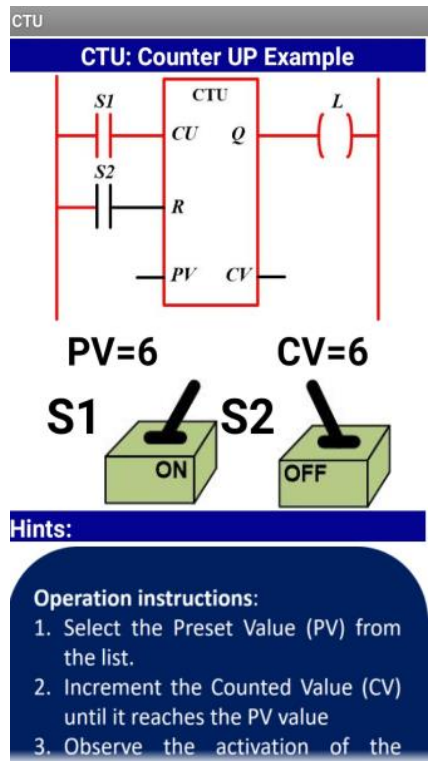

a)
$Q$ is activated?

- What happens to $C V$ when the reset input, $R$, is activated?

- What happens when $C U$ input is activated while $R$ input is on?

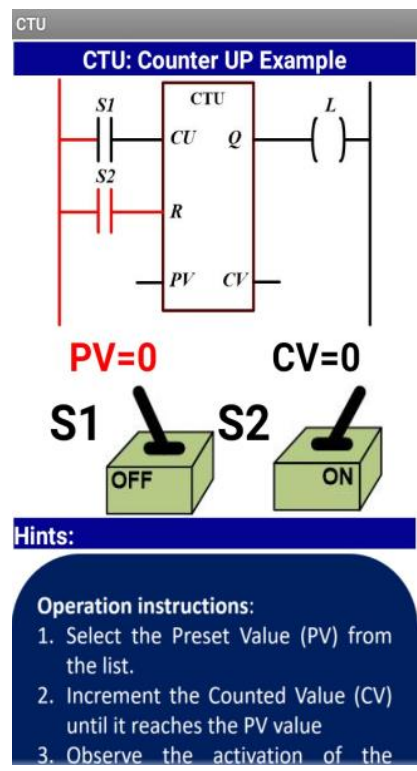

b)

Figure 13: CTU screenshots: a) the counter output is on as $C V=P V$;

b) $S 2$ is switched on resetting the counted value

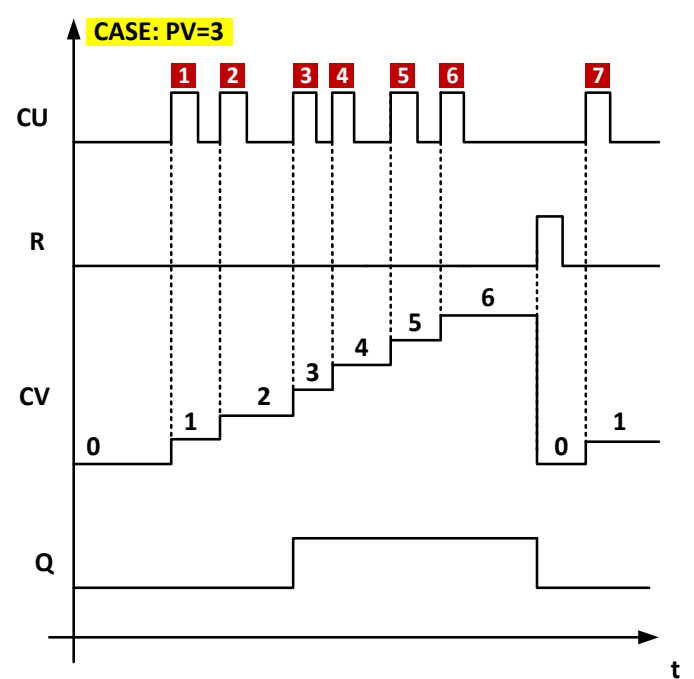

Figure 14: Chronogram example for CTU counter.

The CTD counter can be represented with a generic block diagram as illustrated in Figure 15 a), where: $C D$ represents the counter input, $Q$ the timer output, $L O A D$ the Load input, PV the Preset value and $C V$ the Counted value. A simple LD illustrative example is deployed for the CTD counter as illustrated generically in Figure $15 \mathrm{~b}$ ).

Figure 16 a) presents a TC screenshot related to the CTD counter simulation.
The way the TC simulation for the CTD is programmed, requires the Preset time to be select before the buttons associated with switches $S 1$ and $S 2$ are enabled.

This situation is illustrated by the screenshot presented in Figure 16 a). Moreover, from this figure is also possible to observe that the CTD output is activated when the $C V=0$, which is the initial default situation. Students can follow this particular example simulation also by using the CTD chronogram presented in Figure 18.

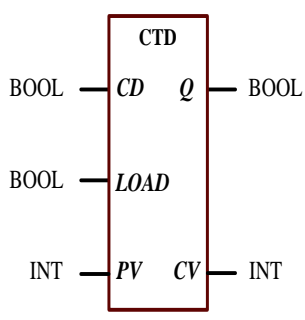

a)

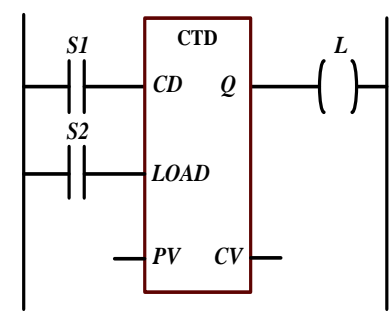

b)
Figure 15: a) General block graphical representation for CTD counter; b) General LD example used for CTD.

The screenshot presented in Figure 16 b) illustrates a situation in which the Counted value was loaded with the value $C V=6$, after the LOAD input, has been activated. 


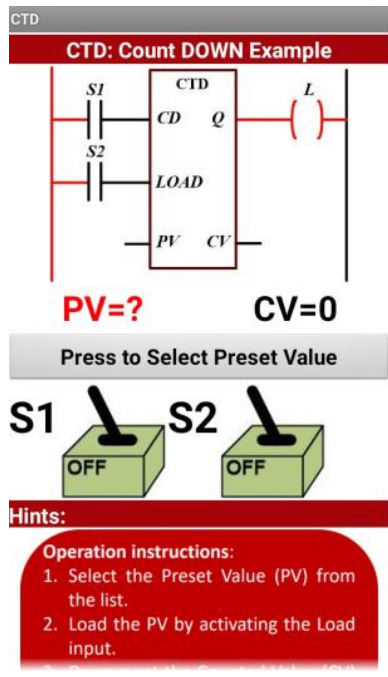

a)
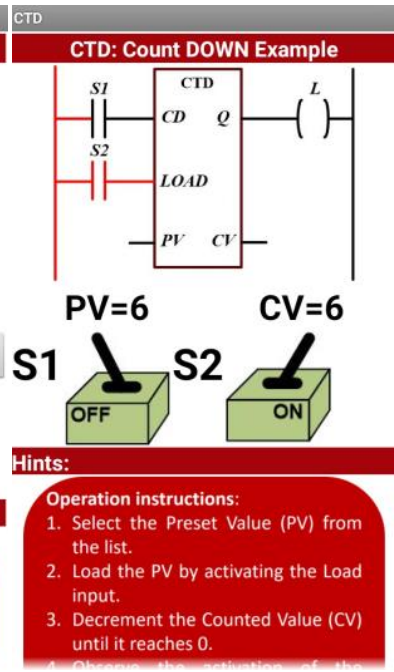

b)
Figure 16: CTD screenshots a) Situation before the

Preset value selected; b) Situation after the Preset value has been selected and the LOAD input activated.

The situation presented in Figure 17 a) represents the case with the $C V$ decreased to 1 . With one more activation of the $C D$ input the $C V$ will reach the 0 value, and the counter output is activated. This situation is illustrated in Figure $17 \mathrm{~b}$ ).

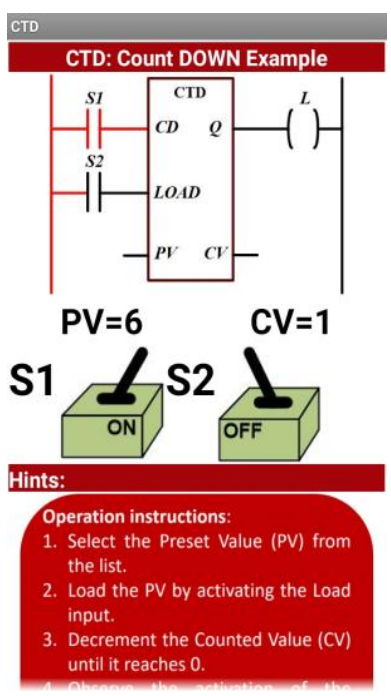

a)
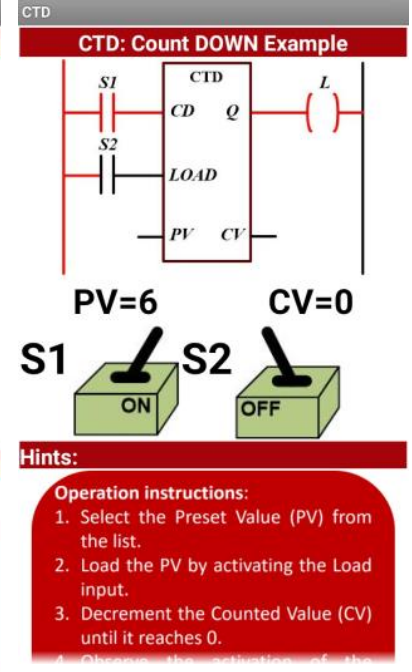

b)
Figure 17: CTD screenshots. a) The CV has been drecerased to 1 ; b) $A s C V=0$ the CTD output, $Q$, is turn on.

After the CTD output activation, when switch $S 1$ is turn off, the Preset value button is made visible allowing the user to select another value. Some CTD specific learning objectives are stated by the following questions:

- What is the CTD output state before the LOAD input is activated?

- What happens to the counter output, $Q$, when the counter output, $C V$, reaches the zero value?
- What happens to $C V$ when the load input, LOAD, is activated before the $C V$ reaches the zero value?

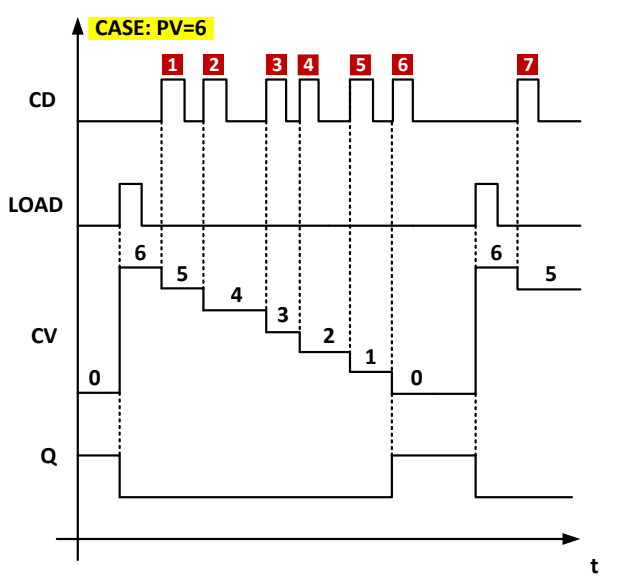

Figure 18: Chronogram example for CTD counter.

The CTUD counter can be represented with a generic block diagram as illustrated in Figure 19 a), where: $C U$ represents the counter increment input, $C D$ represents the counter decrement input, $Q U$ the increment timer output, $Q D$ the decrement timer output, $R$ the Reset input, LOAD the Load input, PV the Preset value and $C V$ the Counted value. A simple LD illustrative example is deployed for the CTUD counter as illustrated generically in Figure 19 b). It is an elementary LD with a single rung, using the following normally open switches: $S 1$ as the counter increment input; $S 2$ as the counter decrement input, S3 as the Reset input, S4 as the LOAD input. The counter increment output, $Q U$, is connected to an output coil, $L 1$, while the counter decrement output. $Q D$, is connected to an output coil, $L 2$.

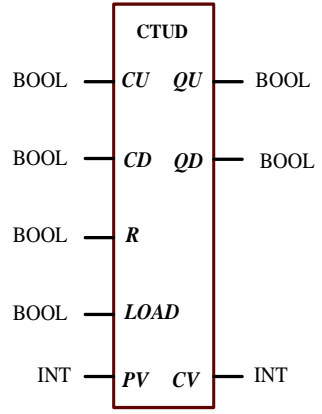

a)

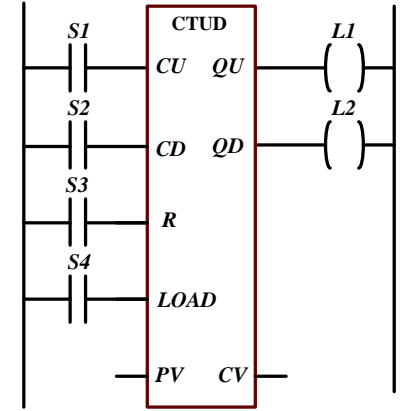

b)
Figure 19: a) General block graphical representation for CTUD counter; b) General LD example used for CTUD.

The screenshot presented in Figure 20 a) illustrates the CTUD default situation prior to the Preset value selection. As it can be seen in this case the decrement output, $C D$, is activated. In the screenshot presented in Figure $20 \mathrm{~b}$ ) the Preset value is $P V=6$ and the Counted value is $C V=2$. 


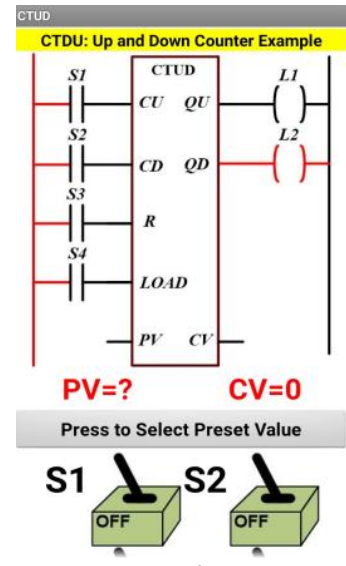

a)

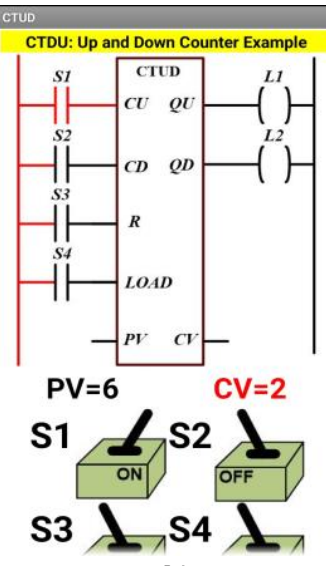

b)

Figure 20: CTUD screenshots. a): Situation before the Preset value selected; b) Situation after the Preset value has been selected and the increment input was activated twice

When the $C V$ reaches the $P V$ the increment output is activated. This case is illustrated by the screenshot presented in Figure 21 a). The screenshot presented in Figure 21 b) illustrates the case when the Reset input is activated.

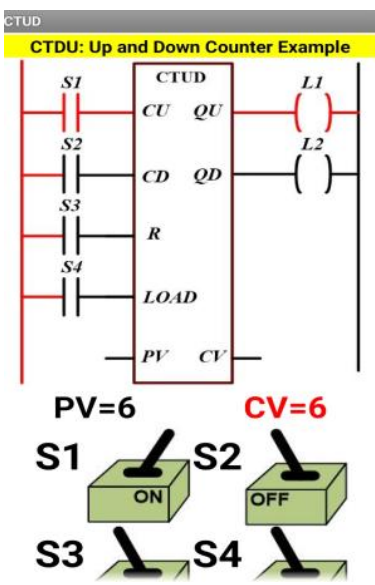

a)
The cases presented in Figures 22 a) and b) illustrate respectively the situations when the Load input is activated and when the Counted value reaches the value $C V=0$. In the last cases the respective counter output is activated.

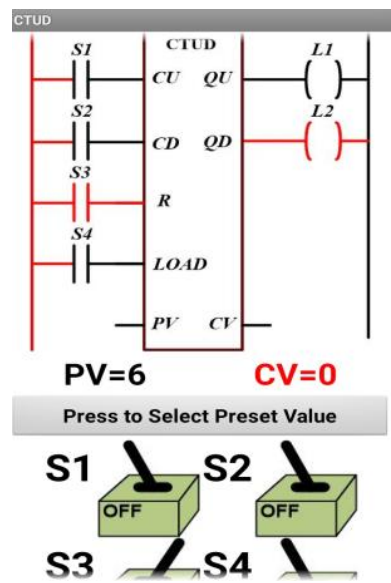

b)

Figure 21: CTUD screenshots a) Situation when the PV is reached; $b$ ) Situation when the Reset, $R$, is activated

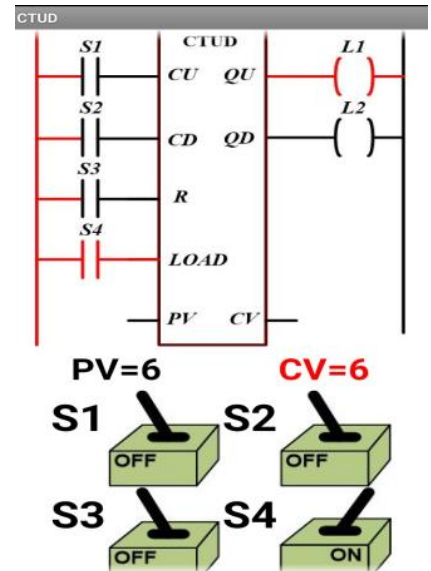

a)
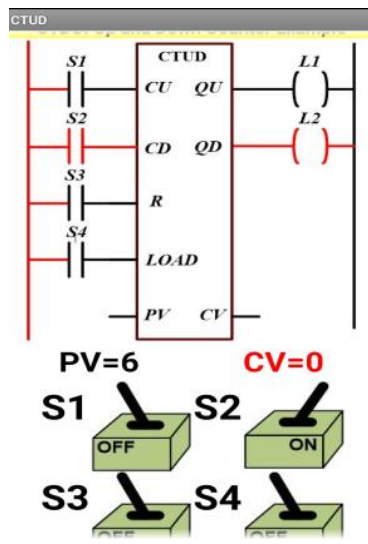

b)

Figure 22: CTUD screenshots a): Situation the LOAD input is activated; b) Situation when CV reaches 0. 
A chronogram example for the CTUD case is provided in TC app, and presented in Figure 23.

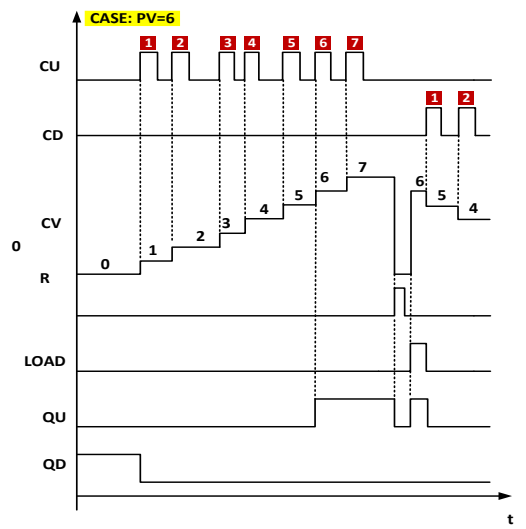

Figure 23: Chronogram example for CTUD counter.

Some CTUD specific learning objectives are stated by the following questions:

- What happens to the increment counter output, $Q U$, when the counter value reaches the $P V$ ?

- What happens to the $C V$ when the counter input $C U$ is activated after the counter output, $Q$ is activated?

- What happens to $C V$ when the reset input, $R$, is activated?

- What happens when the LOAD input is activated?

- What happens when the $C D$ input is activated?

- What happens to the decrement output when $C V=0$ ?

\section{TC use Results and Discussion}

The TC application has been proposed and it currently being used by students of the following curricular units: industrial automation to the 4th year of Electrical and Computers Engineering of UTAD University; 3rd year of Engineering and Industrial Management Master Course of UMinho University, in the first semester of the academic years 2016-2017, 2017-2018 and 2018-2019.

As it was mentioned, the TC App purpose is to be used by students primarily in the out-of-class time, by simulating simulations not fully perceived. However, students should also validate some of the results obtained using the TC App with the physical PLC. An example illustrating the practical validation of the TC simulation example presented for the CTD case (see Figures 16 and 17), the screenshots obtained from the SIEMENS S7-1200, TIA Portal software are presented in Figures 24 and 25.

In Figure 24 a) presents the case before the $L O A D$ input $(L D)$ has been activated loading the Preset value to $P V=6$. Note that this is the default $C T D$ case and the output, $Q$, is activated as $C V=0$. In Figure 24 b) with the activation of $S 2, C V=P T=6$ and the $C T D$ output is switched off. In Figure 25 a) it shown the case after $C V$ has been decreased from $C V=6$ to $C V=1$. One more pulse occurring in $C D$ makes $C V=0$ activating the CTD output. This case is illustrated by Figure $25 \mathrm{~b}$ ).

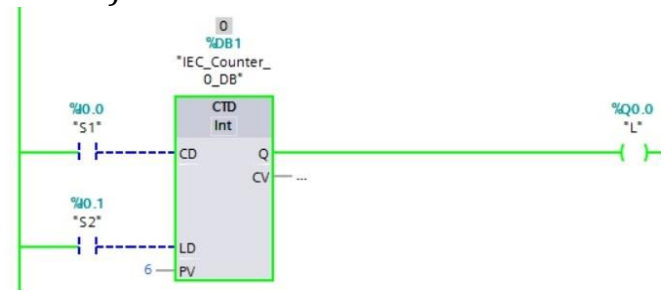

a)

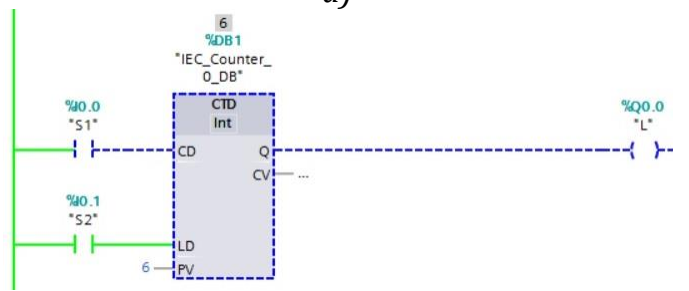

b)

Figure 24: S7-1200 CTD example screenshots: a) Situation before the Preset value selected; b) Situation after the Preset value has been selected with the LOAD input activated.

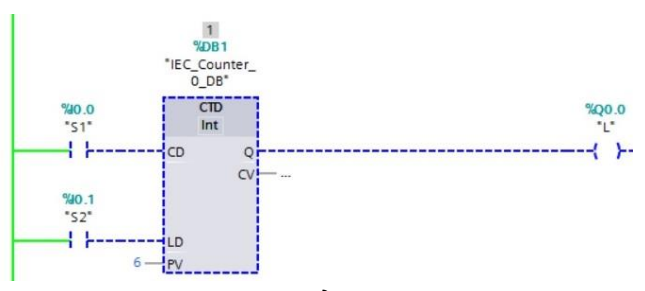

a)

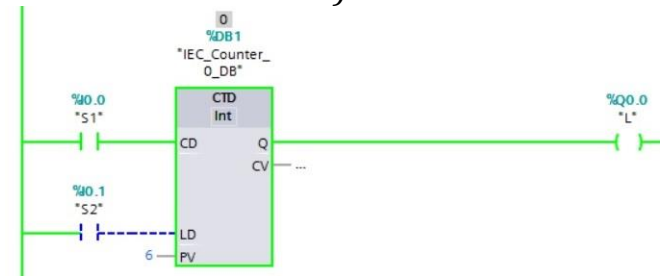

b)

Figure 25: S7-1200 CTD example screenshots: a) The count value was decreased from $C V=6$ to $C V=1 ; b)$ Upon reaching $C V=0$ the counter output is activated.

In the period that $\mathrm{TC}$ has being used, the oral feedback received from students in classes is positive. Students received very well the TC upgrade with the inclusion of the counters components. Suggestions about how to improve the tool has been received as well as to make other applications addressing topics such as shift registers. Also suggestions to include more chronogram examples with different possibilities were received,

\section{Conclusion and Further Work}

In this paper the MIT App-Inventor 2, was reported as a simple to use and effective tool to develop teaching/learning mobile applications for Android based devices. The mobile devices in question are smartphones, and the topics addressed are related to feedback control systems and 
industrial automation. An application entitled TimeCounts (TC) was proposed, addressing both Timers and Counters. Timers and Counters are subjects of the upmost relevance within programmable logic controllers programming languages. The general aim of the proposed application, is to allow students to prior (or after) the practical use of a PLC in classes, use simple simulations of a ladder diagram with the main normalized timers and counters. The timers considered are: on-delay timer, off-delay timer and pulse delay timer. The counters considered are: count up, count down and count up and down. The simulations enable students to perceive specific details regarding both timers and counters operation. Indeed, some timers and counters operation details are difficult to be apprehended by students before simulation and practical PLC testing.

So far, the feedback received from students is quite positive, as well as suggestions to develop more application addressing other PLC programming topics. It is important to state that as the MIT App-Inventor II is (still) an Android operating system tool, this may constitute a disadvantage, as there are students with smartphones using other operating systems. When possible, part of the information included in this paper reported applications is also provided to students in other digital formats such as PDF files.

As future work, a formal pedagogical enquire is going to be proposed, in order to receive more detailed feedback from students. This data is going to be analysed and reported as soon as possible. The potentialities of using MIT-App Inventor II for applications regarding other timers (e.g. on-delay retentive timers), counters and other PLC programming function is going to be explored in the nearby future. Moreover, when fully validated the TC application may be made freely available in the Internet applications repositories (e.g. Google Play Store).

\section{References}

[1] Drath R. and Horch A. (2014), Industrie 4.0 - Hit or Hype?, In IEEE Industrial Electronics Magazine 01/2014; 8(2):56-58.

[2] Da Xu L., Xu E. L. and Li L. (2018), Industry 4.0: state of the art and future trends, International Journal of Production Research, Vol. 56, No. 8, 2941-2962.

[3] Stock T. and Seliger G., (2016), Opportunities of Sustainable Manufacturing in Industry 4.0, Procedia CIRP 40, 536 - 541.

[4] Colombo a. W., Karnouskos S., Shi Y., Yin S. and Kaynak O. (2016), Industrial Cyber-Physical Systems, Proceedings of the IEEE, Vol. 104, No. 5.

[5]IEC, (2018), International Electrotechnical Commission, http://www.iec.ch/, Last retrieved 2018/2/1.
[6] Heinz K. J. and Tiegelkamp M., (2010), IEC 611313: Programming Industrial Automation Systems. Springer.

[7] Vyatkin V., (2009), “The IEC 61499 Standard and its Semantics- Bridging the Gap Between PLC Programming Languages and Distributed Systems", IEEE Ind. Electron. Mag., Vol 3. No. 4, pp. 40-48.

[8] Roschelle, J. (2003). Keynote paper: Unlocking the learning value of wireless mobile devices. Journal of Computer Assisted Learning, 19(3), pp. 260-272.

[9] Georgiev, T., Georgieva, E., and Trajkovski, G. (2006). Transitioning from e-learning to $\mathrm{m}$ learning: Present issues and future challenges. In Software Engineering, Artificial Intelligence, Networking, and Parallel/Distributed Computing, 7th ACIS Int. Conf., pp- 349-353.

[10] So, S. (2016). Mobile instant messaging support for teaching and learning in higher education. Internet and Higher Education, 31, pp. 32-42.

[11] MITApp2 (2018a). MIT App Inventor. About page http://www.appinventor.org/about, last retrieved 07.11.2018

[12] Pokress S. C. and Veiga J. J. D., (2013). MIT App Inventor, Enabling personal mobile computing. PROMOTO '13, ACM, Indianapolis, USA, October 26.

[13] Roy K., (2015). Position Statement: App Inventor Instructional Resources for Creating Tangible Apps. IEEE Blocks and Beyond Workshop, pp. 119-120.

[14] Moura Oliveira, P. B., (2015). Teaching automation and control with App Inventor applications. In Global Engineering Education Conference EDUCON, Tallinn, IEEE, 879-884.

[15] Soares F., Oliveira P.M. and Leão C. P., (2016). Control Engineering Learning by Integrating AppInventor Based 12th Portuguese Conference on Automatic Control, Vol. 402, pp. 845-855.

[16] Moura Oliveira P.B, Boaventura Cunha J. and Soares F. (2019), Integrating MIT App-Inventor in PLC Programming Teaching, J. Machado et al. (Eds.): HELIX 2018, Springer, LNEE 505, pp. 1724.

[17] Yao-Ting S., Kuo-En C. and Liu Tzu-Chien (2016), The effects of integrating mobile devices with teaching and learning on students' learning performance: A meta-analysis and research synthesis, Computers \& Education 94, pp. 252e75.

[18] MITApp2 (2018b). MIT App Inventor. Tutorials http://appinventor.mit.edu/explore/ai2/tutorials, Last retrieved 07.11.2018.

[19] Siemens S7-1200, (2018): http://w3.siemens.com/mcms/programmable-logiccontroller/en/basic-controller/s71200/pages/default.aspx, Last retrieved 07.11.2018. 